\section{Comment on "Search for Axionlike Dark Matter with a Liquid-State Nuclear Spin Comagnetometer"}

This elegant experiment [1] searched for axionic dark matter "wind" using two different nuclear magnetometers in the same molecule to cancel magnetic effects. However, the authors used a complicated analysis of the power spectra (described in Supplemental Material) to obtain the constraint shown in the left panel of their Fig. 3, which is clearly flawed. Any constraint on oscillations with periods, $\tau$, long compared to the span of the data, $T=2.642 \times 10^{6} \mathrm{~s}$, cannot be tighter than those on oscillations with periods shorter than $T$ and, in fact, must be substantially weaker. This reduction in experimental sensitivity can be seen in other recent works that searched for similar effects [2-5].

A simple analysis that provides the correct result and quantitative insight is a linear least-squares analysis such as that used in Ref. [5]. Here one fits the time-series data and its errors with orthogonal, typically nonlinear, basis functions that model the data expected from the physics being probed. In the present case, for each assumed axion mass there will be two such functions of time that contain the nonlinear transforms between celestial and laboratory coordinates multiplied by $\sin (2 \pi t / \tau)$ or $\cos (2 \pi t / \tau)$, where $t$ is the time of the measurement. At each assumed axion frequency, one makes a simultaneous linear fit of the data to the two basis states, obtaining two fit amplitudes and their errors along with the correlation matrix. Oscillation constraints marginalized over the axion phase are found by combining the quadrature (sine and cosine) amplitudes. When $\xi=\tau / T<1$, the two quadrature basis functions have virtually similar mean magnitudes and are nearly orthogonal. The resulting constraint of the marginalized amplitude is (barring a real signal and assuming $\delta \ll \tau$, where $\delta$ is the duration of an individual measurement) nearly independent of the frequency. However, when $\xi>1$, the basis states either are highly anticorrelated or now have very different magnitudes. This greatly increases the uncertainties in the marginalized amplitude. As a concrete example, consider the case where the zero of the time is arbitrarily set to zero at the center of the data span. Now the basis functions are proportional to $\sin (2 \pi t / \tau) \approx 2 \pi t / \tau$ and $\cos (2 \pi t / \tau) \approx 1-(2 \pi t / \tau)^{2} / 2$, where $-T / 2<t<+T / 2$. The central value and error of the extracted sine amplitude blow up as $\tau /(\pi T)$, which for oscillations corresponding to the left-hand edge of Fig. 3 has a value of 6.0. This argument is quite general and does not depend on a choice

Published by the American Physical Society under the terms of the Creative Commons Attribution 4.0 International license. Further distribution of this work must maintain attribution to the author(s) and the published article's title, journal citation, and DOI. of the origin of the timescale. For example, suppose that the origin of time were set so that the phase of the axion signal was $\pi / 4$ at the midpoint of the data span. Now neither of the two basis functions tends to zero, but the two functions are almost perfectly anticorrelated, giving essentially the same blowup factor in the extracted marginalized amplitude.

We trace the strange long-period behavior in Fig. 3, where the constraint actually becomes tighter as $\pi T / \tau<2.5$, to the discussion in derivation of the constraint level [Eqs. (S12)-(S17)] in Supplemental Material. These relate the data $\Delta \mathcal{R}$ to the axion coupling $g_{a N N}$ and the phase $\phi$ of the axion oscillation, with $g_{a N N}$ proportional to $\Delta \mathcal{R} / \sin \phi$. The authors account for the effect on $g_{a N N}$ of the unknown phase by averaging $|\sin \phi|$ over the interval for $\phi$ between 0 and $2 \pi$. This is incorrect, as the constraint is actually proportional to $1 / \sin \phi$. (Note that approaches where the $\phi$ is a free parameter will not have a pole for any nonzero frequency.) Assuming that the analysis for signals with $\tau<T$ is correct, the exclusion limit at the left-hand edge of Fig. 3 should be 9.5 times higher than shown. This would be $\sim 15 \%$ less constraining than the previous limit set using neutron EDM data. The authors cite Ref. [6] as containing a corrected figure. The current version (v3) of Ref. [6] still has a problem, as the low-frequency scaling of Fig. 4 does not match the analytic form that we derived in our Comment. Figures 3 and 5, which show other experiments, have the correct scaling.

This work was supported in part by National Science Foundation Grants No. PHY-1305726 and No. PHY1607391.

\author{
E. G. Adelberger ${ }^{1, *}$ and W. A. Terrano ${ }^{2, \dagger}$ \\ ${ }^{1}$ Center for Experimental Nuclear Physics and Astrophysics, \\ Box 354290, University of Washington \\ Seattle, Washington 98195-4290, USA \\ ${ }^{2}$ Department of Physics, Princeton University \\ Princeton, New Jersey 08550, USA
}

Received 27 June 2019; published 14 October 2019

DOI: 10.1103/PhysRevLett.123.169001

*eadelberger@gmail.com

†terrano@princeton.edu

[1] T. Wu et al., Phys. Rev. Lett. 122, 191302 (2019).

[2] K. Van Tilburg, N. Leefer, L. Bougas, and D. Budker, Phys. Rev. Lett. 115, 011802 (2015).

[3] A. Hees, J. Guéna, M. Abgrall, S. Bize, and P. Wolf, Phys. Rev. Lett. 117, 061301 (2016).

[4] C. Abel et al., Phys. Rev. X 7, 041034 (2017).

[5] W. A. Terrano, E. G. Adelberger, C. A. Hagedorn, and B. R. Heckel, Phys. Rev. Lett. 122, 231301 (2019).

[6] G. P. Centers et al., arXiv:1905.13650v3. 